\title{
Application and Comparison of Uncertainty Quantification Methods for Railway Vehicle Dynamics with Random Mechanical Parameters
}

\author{
Dawei ZHANG*, Peijuan XU**, Daniele BIGONI*** \\ *School of Automobile, Chang'an University, Xi'an 710064, China, E-mail: dwzhang@chd.edu.cn \\ **School of Highway, Chang'an University, Xi'an 710064, China, E-mail: p_xu_0428@163.com \\ ***Department of Aeronautics and Astronautics, Massachusetts Institute of Technology, Cambridge MA 02139, USA, \\ E-mail:dabi@mit.edu \\ crossref http://dx.doi.org/10.5755/j01.mech.25.6.23278
}

\section{Introduction}

Deterministic simulations are insufficient to represent the whole physics of the vehicle-track coupled system, which contains several kinds of variabilities, i.e., variability of mechanical parameters related to the vehicle mass, stiffness and damping of different suspensions, variability of contact parameters of the wheel-rail interaction and variability of the track design and quality [1]. Those variabilities play an important role in the safety, ride quality, and assessment criteria of running trains. When using the simulation for assessment purposes, it is therefore crucial to consider those uncertain variabilities and utilize uncertainty quantification to investigate uncertainties in dynamical systems.

In past decades, generalized polynomial chaos theory, a kind of uncertainty quantification, has been widely adopted to model complex nonlinear dynamic systems operating in the presence of parametric and external uncertainties [2, 3]. For road vehicles, Kewlani et. al. [4] presented polynomial chaos approaches explicitly considering parametric uncertainty during the model formulation. Wu et. al. $[5,6]$ proposed a Polynomial-Chaos-Chebyshev Interval method for vehicle dynamics involving hybrid uncertainty parameters and a flexible multibody system with uncertain material properties was considered as well.

With regard to the railway system, full-scale measurements have shown that stochastic variations in the track structure is inevitable [7, 8]. For example, railway track stiffness variations were found and studied [9]. As for the source of uncertainties, Funfschilling et. al. [1, 10] addressed that the mechanical characteristics can be different among vehicles, due to difference in numbers of passengers or mass of goods, process uncertainties and the components. By considering the random distribution of the vehicle and track parameters, some papers focused on the uncertainty qualification in the vehicle-track coupled system. Bigoni et. al. [11] paid attention to the critical speed of a bogie and its sensitivity analysis.

In addition, uncertainties of operation environment for railway vehicles (called external uncertainties) are essential factors for stochastic dynamic behaviors. Perrin et. al. [12] built a stochastic model for track geometry based on a spatial and statistical decomposition and analyzed the influence of the track geometry variability on the train dynamics. To visualize the long-term evolution of the stability and the comfort of the train, Lestoille et. al. [13] formulated a stochastic model of the track geometry to identify track irregularities for dynamics of high-speed trains. Recently, Xu and Zhai $[14,15]$ proposed a computational model to analyze the temporal-spatial stochastic vibrations of vehicletrack systems and applied the probability density evolution method to solve the de-livery problem of probabilities between excitation inputs and response outputs.

Previous studies have indicated that uncertainties lead to a significant influence on the dynamic behavior of a simple or complex system. However, current outcomes on how uncertainties in railway vehicle-track system per-form in terms of vehicle performance assessment is still limited. In order to shed light on uncertainties in the vehicle-track system, this paper begins by outlining the uncertainty qualification methods, including Sampling-based method, stochastic Gelerkin method and stochastic Collocation method in Section 2. The following sections set out the applications of uncertainty quantification methods in railway vehicle dynamics with parametric uncertainties in detail. Uncertainties are modelled as independent random inputs with Gaussian distributions. A comparison of three methods is carried out in terms of the computational accuracy and efficiency in Section 3, where an SDOF wheel model with two random parameters is established. Then, a simplified TDOF bogie model with three suspension parameters is constructed in Section 4 to analyze the critical speed through SCM and LHS. At last, the focus of Section 5 is to investigate the uncertainties on the impact behavior under a rail defect and dynamic response under a track irregularity by using a vertical vehicle-track coupled model with parametric uncertainties on the basis of SCM.

\section{Uncertainty qualification method}

To illustrate how to evaluate the uncertainty qualification of dynamic systems with random input parameters, a simple single-degree-of-freedom system (SDOF) is firstly taken as an example. For this purpose, the equation of motion for a simple SDOF system is formulated directly by expressing the equilibrium of all forces acting on the mass according to the d'Alembert's principle, which is found to be:

$$
\left\{\begin{array}{c}
A_{1} \ddot{y}(t)+A_{2} \dot{y}(t)+A_{3} y(t)=A_{4} \\
y(0)=A_{5}
\end{array},\right.
$$

where: $y(t)$ means the time-varying displacement. In general, $A_{i}$ is constant for a deterministic system. If random parameters are considered, Eq. (1) becomes a stochastic differential equation, in which $A_{i}$ represents a function of a random vector $\mathbf{x}$ defined as, 


$$
A_{i}=f_{i}(\mathbf{x}), i=1 \sim n,
$$

where: $\mathbf{x}=\left(x_{1}, \ldots, x_{d}\right)$ is an random vector with $d$ dimensions.

The stochastic solution of the SODF system is now assumed to be $y=g(t, \mathbf{x})$. Hence, the mean and the variance of this solution can be defined by:

$$
\begin{aligned}
& \mu(t)=E[y(t)]=\int_{\Omega^{d}} g(t, \mathbf{x}) \rho(\mathbf{x}) d \mathbf{x}, \\
& \sigma^{2}(t)=V[y(t)]=\int_{\Omega^{d}}[g(t, \mathbf{x})-\mu(t)]^{2} \rho(\mathbf{x}) d \mathbf{x},
\end{aligned}
$$

where: $\rho(\mathbf{x})$ is the probability density function (PDF) of the random vector $\mathbf{x}$ and the integrals are computed over its domain integration $\Omega^{d}=\prod_{i=1}^{d}\left[a_{i}, b_{i}\right]=\left[a_{1}, b_{1}\right] \times \ldots \times\left[a_{d}, b_{d}\right]$; $E(\cdot)$ and $V(\cdot)$ are the expectation and variance operators respectively; $\sigma$ represents the standard deviation $(\mathrm{Std}) ; \sigma / \mu$ denotes the coefficient of variation $(\mathrm{CoV})$, which is commonly used to assess the dispersion degree of samples. Similarly, the method is also applicable for a multi-degree-of-freedom system.

In the following sections, three common methods to realize the stochastic analysis are discussed in detail, i.e., sampling-based method, stochastic Galerkin method and stochastic Collocation method.

1. Sampling-based method.

A straightforward technique is to estimation the integral in Eqs. (3) and (4). Final expressions are shown as

$$
\begin{aligned}
& \mu(t) \approx \frac{1}{M} \sum_{j=1}^{M} g\left(t, \mathbf{x}^{j}\right), \\
& \sigma^{2}(t) \approx \frac{1}{M-1} \sum_{j=1}^{M}\left[g\left(t, \mathbf{x}^{j}\right)-\mu(t)\right]^{2},
\end{aligned}
$$

where: $\mathbf{x}^{j}$ is sample data, which are generally acquired based on Monte Carlo method (MC) and Latin Hypercube Sampling (LHS).

2. Stochastic Galerkin method.

The so-called stochastic Galerkin method (SGM) $[16,17]$ is a kind of classical polynomial chaos, where orthogonal polynomials are used to represent solutions. For Gaussian random variables, Probabilists' Hermite polynomial is an orthogonal system with respect to the measure $\rho$ defined by the standard normal distribution $\rho(x)=\frac{1}{\sqrt{2 \pi}} e^{-\frac{x^{2}}{2}}$,

$$
\left\{\begin{array}{c}
\mathrm{H}_{n+1}(x)=x H_{n}(x)-n \mathrm{H}_{n-1}(x), n>0 \\
\mathrm{H}_{0}(x)=1 \\
\mathrm{H}_{1}(x)=x
\end{array} .\right.
$$

The above orthogonality relations should hold:

$$
\begin{aligned}
& \int_{-\infty}^{\infty} \mathrm{H}_{m}(x) \mathrm{H}_{n}(x) d \rho(x)= \\
& \int_{-\infty}^{\infty} \mathrm{H}_{m}(x) \mathrm{H}_{n}(x) \frac{1}{\sqrt{2 \pi}} e^{-\frac{x^{2}}{2}} d x=\gamma_{n} \delta_{n m}=n ! \delta_{n m},
\end{aligned}
$$

where: $\delta_{m n}$ is the Kronecker delta function, herewith $\delta_{m n}=0$ if $m \neq n$ and $\delta_{m n}=1$ if $m=n$; and $\gamma_{n}$ is a positive constant often termed by a normalization constant, i.e., $\gamma_{n}=n$ !.

Thus, the considered set of basis $\left\{\mathrm{H}_{\mathbf{k}}(\mathbf{Z})\right\}_{|\mathbf{k}| \leq N}$, where $\mathbf{k}$ is a multi-index based on graded lexicographic ordering. Then the random input parameters and the solution can be reformed as:

$$
\begin{aligned}
& A_{i}(\mathbf{x}) \approx \sum_{|\mathbf{k}|=0}^{N} \hat{A}_{i, \mathbf{k}} \mathrm{H}_{\mathbf{k}}(\mathbf{x}), \\
& g(t, \mathbf{x}) \approx \sum_{|\mathbf{k}|=0}^{N} \hat{g}_{\mathbf{k}} \mathrm{H}_{\mathrm{k}}(\mathbf{x}) .
\end{aligned}
$$

The Galerkin procedure results in:

$$
\begin{gathered}
\left\{\begin{array}{c}
E\left[\left(A_{1} \ddot{y}+A_{2} \dot{y}+A_{3} y\right) \mathrm{H}_{\mathbf{k}}(\mathbf{x})\right]=E\left[A_{4} \mathrm{H}_{\mathbf{k}}(\mathbf{x})\right], \\
\hat{g}_{\mathbf{k}}(0)=\hat{g}_{0, \mathbf{k}}
\end{array}\right. \\
\hat{g}_{\mathbf{k}}=\frac{1}{\gamma_{\mathbf{k}}} \int_{\Omega^{d}} g(t, \mathbf{x}) \mathrm{H}_{\mathbf{k}}(\mathbf{x}) \rho(\mathbf{x}) d \mathbf{x},
\end{gathered}
$$

where: $\hat{A}_{i, \mathbf{k}}$ and $\hat{g}_{\mathbf{k}}$ are the expansion coefficients for the random inputs and outcomes respectively. Hence, the mean and Std are solved as:

$$
\begin{aligned}
& \mu(t) \approx \hat{g}_{0}(t), \\
& \sigma^{2}(t) \approx \sum_{|\mathbf{k}|=0}^{N} \gamma_{\mathbf{k}} \hat{g}_{\mathbf{k}}^{2}(t) .
\end{aligned}
$$

3. Stochastic Collocation method.

Collocation method [16] is the so-called pseudo spectral approach [17], which consists in the construction of a discrete projection of the quantity of interest. To get the solution of Eq. (11), a simple tensor product structured Gauss cubature rules [11] is used to define points and weights. The $d$-dimensional Gauss quadrature points and weights are denoted by $\left\{\mathbf{x}^{j}, \mathbf{w}^{j}\right\}_{j=1}^{M}$. Then expansion coefficients can be refined as:

$$
\begin{aligned}
& \hat{g}_{\mathbf{k}}=\frac{1}{\gamma_{\mathbf{k}}} \int_{\Omega^{d}} g(t, \mathbf{x}) \mathrm{H}_{\mathbf{k}}(\mathbf{x}) \rho(\mathbf{x}) d \mathbf{x} \approx \\
& \approx \frac{1}{\gamma_{\mathbf{k}}} \sum_{j=1}^{M} g\left(t, \mathbf{x}^{j}\right) \mathrm{H}_{\mathbf{k}}\left(\mathbf{x}^{j}\right) \mathbf{w}^{j} .
\end{aligned}
$$

Substituting Eq. (15) into Eqs. (13) and (14), the mean and Std can be obtained.

\section{SODF wheel model}

In this section, a SODF wheel model in Fig. 1 is given as an example to illustrate how to realize the solution of dynamics equations by means of SGM.

The equation of motion of the SDOF wheel model is built as follows:

$$
m \ddot{q}+C \dot{q}+K q=0,
$$


where: $m, K$ and $C$ represent the wheel mass, spring stiffness and damping coefficient respectively. Among those, $K$ and $C$ are two random input parameters distributed normally according to $K \sim N\left(\mu_{s}, \sigma_{s}{ }^{2}\right)$ and $C \sim N\left(\mu_{c}, \sigma_{c}^{2}\right)$. The corresponding Galerkin basis will be the Probabilists' Hermite polynomials. Then, approximations of random input parameters can be expressed as:

$$
\begin{aligned}
& K\left(x_{1}\right)=\sum_{i=0}^{N} \hat{k}_{i} H_{i}\left(x_{1}\right)=\mu_{s}+\sigma_{s} x_{1}, \\
& C\left(x_{2}\right)=\sum_{i=0}^{N} \hat{c}_{i} H_{i}\left(x_{2}\right)=\mu_{c}+\sigma_{c} x_{2} .
\end{aligned}
$$

Similarly, the solutions of the displacement, velocity and acceleration for the single wheel can be formulated as:

$$
\begin{aligned}
& q_{N}\left(t, x_{1}, x_{2}\right)=\sum_{|\mathbf{i}|=0}^{N} \hat{q}_{\mathbf{i}}(t) H_{\mathbf{i}}\left(x_{1}, x_{2}\right), \\
& \dot{q}_{N}\left(t, x_{1}, x_{2}\right)=\sum_{|\mathbf{i}|=0}^{N} \dot{\hat{q}}_{\mathbf{i}}(t) H_{\mathbf{i}}\left(x_{1}, x_{2}\right), \\
& \ddot{q}_{N}\left(t, x_{1}, x_{2}\right)=\sum_{|\mathbf{i}|=0}^{N} \ddot{\hat{q}}_{\mathbf{i}}(t) H_{\mathbf{i}}\left(x_{1}, x_{2}\right),
\end{aligned}
$$

where: $\mathbf{i}$ is a multi-index, $\mathbf{i}=\left(i_{1}, i_{2}\right)$ with $|\mathbf{i}|=i_{1}+i_{2}$. Then 2 variate Galerkin basis functions are the products of the univariate polynomials, i.e., $H_{\mathbf{i}}\left(x_{1}, x_{2}\right)=H_{i_{1}}\left(x_{1}\right) H_{i_{2}}\left(x_{2}\right)$.The Galerkin procedure results in:

$$
\begin{aligned}
& E\left[m \frac{d^{2} q_{N}}{d t^{2}} H_{\mathbf{k}}\left(x_{1}, x_{2}\right)\right]+ \\
& +E\left[k_{N} q_{N} H_{\mathbf{k}}\left(x_{1}, x_{2}\right)+c_{N} \frac{d q_{N}}{d t} H_{\mathbf{k}}\left(x_{1}, x_{2}\right)\right]=0,
\end{aligned}
$$

where: $\mathbf{k}=0, \ldots, N$.

Upon substituting Eqs. (17)-(21) in Eq. (22), Eq. (22) can be rewritten as:

$$
\begin{aligned}
& m \ddot{\hat{q}}_{\mathbf{k}}=-\mu_{s} \hat{q}_{\mathbf{k}}- \\
& -\frac{\sigma_{s}}{\gamma_{\mathbf{k}}} \sum_{\mathbf{j} \mathbf{j}=0}^{N} \hat{q}_{\mathbf{j}} E\left[H_{1}\left(x_{1}\right) H_{\mathbf{j}}\left(x_{1}, x_{2}\right) H_{\mathbf{k}}\left(x_{1}, x_{2}\right)\right]- \\
& -\mu_{c} \dot{\hat{q}}_{\mathbf{k}}-\frac{\sigma_{c}}{\gamma_{\mathbf{k}}} \sum_{\mathbf{j} \mathbf{j}=0}^{N} \dot{\hat{q}}_{\mathbf{j}} E\left[H_{1}\left(x_{2}\right) H_{\mathbf{j}}\left(x_{1}, x_{2}\right) H_{\mathbf{k}}\left(x_{1}, x_{2}\right)\right],
\end{aligned}
$$

where: $\gamma_{\mathbf{k}}=E\left[H_{\mathbf{k}}\left(x_{1}, x_{2}\right) H_{\mathbf{k}}\left(x_{1}, x_{2}\right)\right]=\gamma_{k_{1}} \gamma_{k_{2}}=k_{1} ! k_{2} !$,

$$
E\left[H_{1}\left(x_{1}\right) H_{\mathbf{j}}\left(x_{1}, x_{2}\right) H_{\mathbf{k}}\left(x_{1}, x_{2}\right)\right]=e_{1 j_{1} k_{1}} \gamma_{k_{2}} \text {, }
$$$$
E\left[H_{1}\left(x_{2}\right) H_{\mathbf{j}}\left(x_{1}, x_{2}\right) H_{\mathbf{k}}\left(x_{1}, x_{2}\right)\right]=\gamma_{k_{1}} e_{1 j_{2} k_{2}} \text {, }
$$$$
e_{i j k}=\frac{i ! j ! k !}{(s-i) !(s-j) !(s-k) !},
$$

$s \geq i, j, k$, and $2 s=i+j+k$ is even.

Above parameters in Eq. (23) are constants evaluated in advance before the computation of the model.

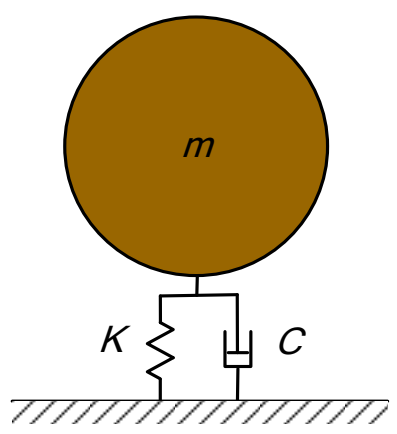

Fig. 1 SODF wheel model

As mentioned above, the SGM procedure for the SDOF wheel model is formulated. In addition, further validation for solutions is proceeded by LHS and SCM. In terms of LHS, 1000, 10,000 and 50,000 points are tested respectively, while for the simulation process of SCM, two-dimensional nodes (6 nodes in each dimension) are generated based on the Gauss quadrature rule.

The parameters of the SDOF wheel system refer to: $m=10 \mathrm{~kg} ; K \sim N\left(100 \mathrm{~N} / \mathrm{m}, 10^{2}(\mathrm{~N} / \mathrm{m})^{2}\right) ; C \sim N(20 \mathrm{~N} \cdot \mathrm{s} / \mathrm{m}$, $\left.2^{2}(\mathrm{~N} \cdot \mathrm{s} / \mathrm{m})^{2}\right) ; y(0)=0.1 \mathrm{~m}$. The numerical results solved by SGM with a polynomial order of $N=6$ is shown in Fig. 2, where stochastic responses of the vertical displacement varying with time are illustrated. In Fig. 2 a, the solid line represents the mean of the vertical displacement and the other

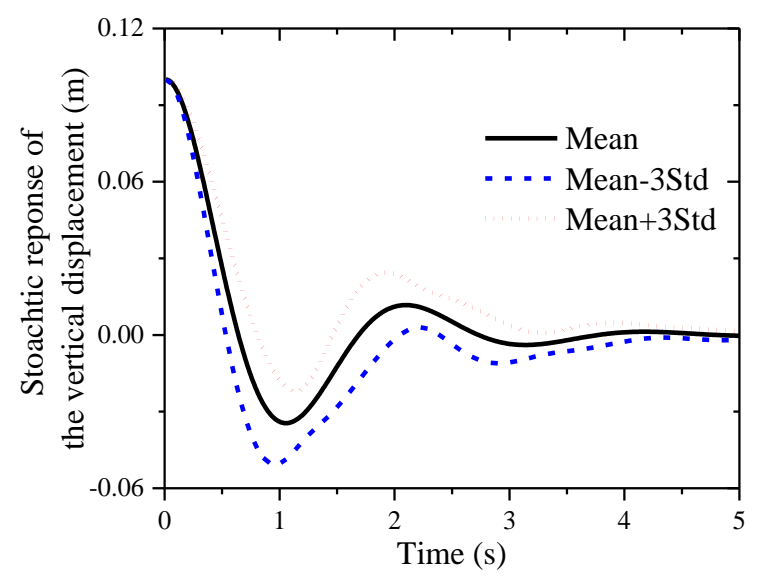

a

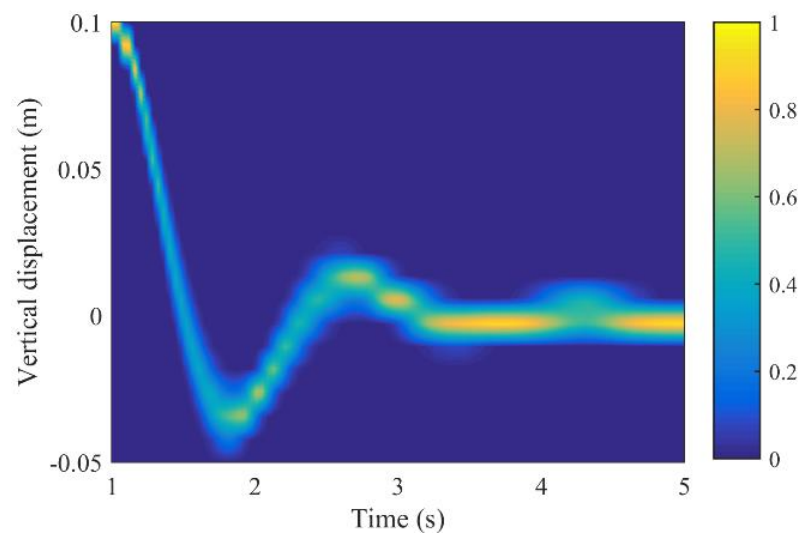

b

Fig. 2 Stochastic responses of the vertical displacement in the case of initial displacement of $0.1 \mathrm{~m}$ : (a) probable boundary region and (b) PDF evolution of vertical displacement varying with time 
two dashed lines donate values of $\mu+3 \sigma$ and $\mu-3 \sigma$ respectively. The zone between two dashed lines is the possible range of the vertical displacement. The relatively large range demonstrates that the random input indeed obviously influences the vertical wheel displacement. In the other words, an obvious uncertainty of the vertical wheel displacement is caused by the random input parameters. In addition, PDF evolution of the displacement is given in Fig. 2 $\mathrm{b}$, where the probability of the displacement at each time $t$ can be observed. The area labelled in yellow indicates the largest probability of the displacement locating.

Furthermore, comparisons of result differences between LHS and SGM are shown in Fig. 3 respectively, in terms of mean and Std. As for those dash lines, it is evident that the more tested points of LHS are, the smaller difference is, both in Figs. 3 a and b. Thus, the results with larger amount of sample inputs in LHS are more similar with the results computed by SGM. However, it should be noted that larger amount of sample data inevitably leads to the increase of computational cost. Therefore, SGM shows its strength in terms of efficiency and computational cost. On the other hand, it is difficult and cumbersome for Galerkin method to deal with a simple or complex system since this method needs to be derived in detail in spite of the existing wellbuilt model. Conversely, the SCM can be easily adopted to solve the complexity or nonlinearity of the original problem, once a reliable deterministic solver is developed. To this end, the difference between the results of SGM and SCM are also compared. As mentioned above, 36 nodes are tested in SCM. It can be obviously seen that differences can be negligible and results of SGM and SCM are in a quit good agreement.

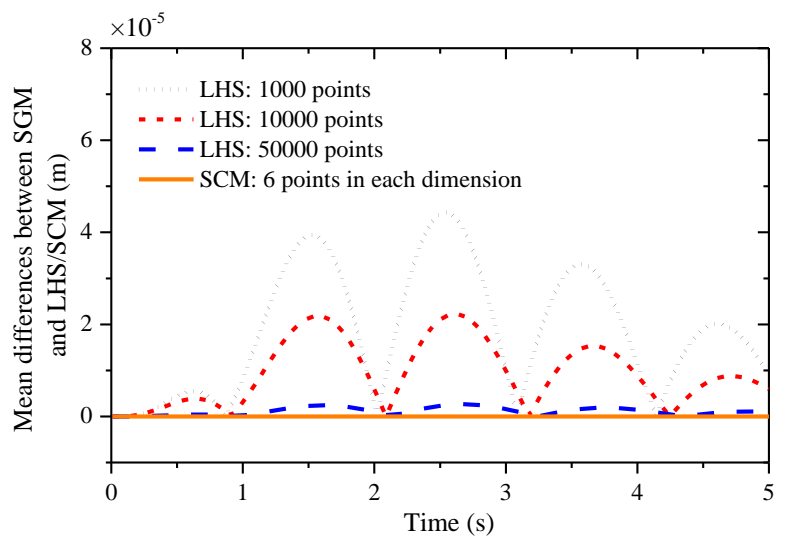

a

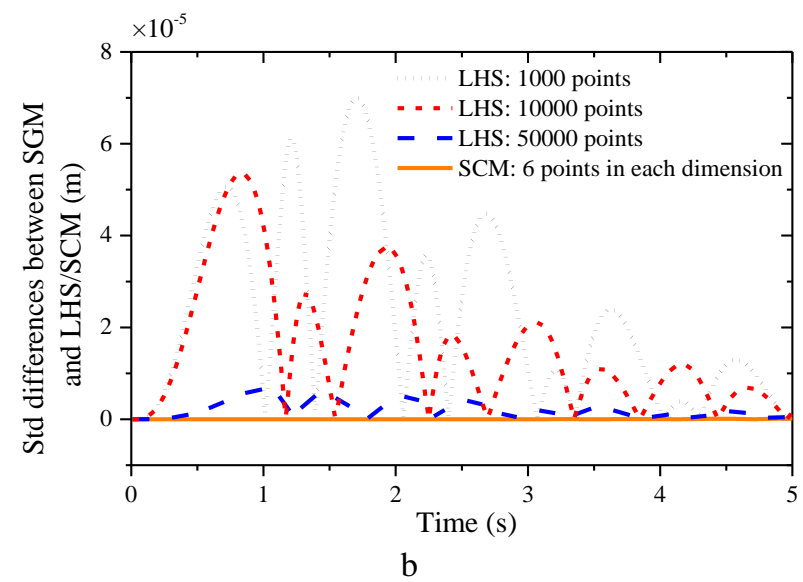

Fig. 3 Differences between SGM and LHS/SCM: (a) mean and (b) Std

\section{TDOF Bogie Model}

In this section, a two-degree-of-freedom model (TDOF) for a railway bogie is established to study the critical speed with random input. As shown in Fig. 4, the Cooperrider bogie model [18] is adopted, where only lateral and yaw motions are considered.

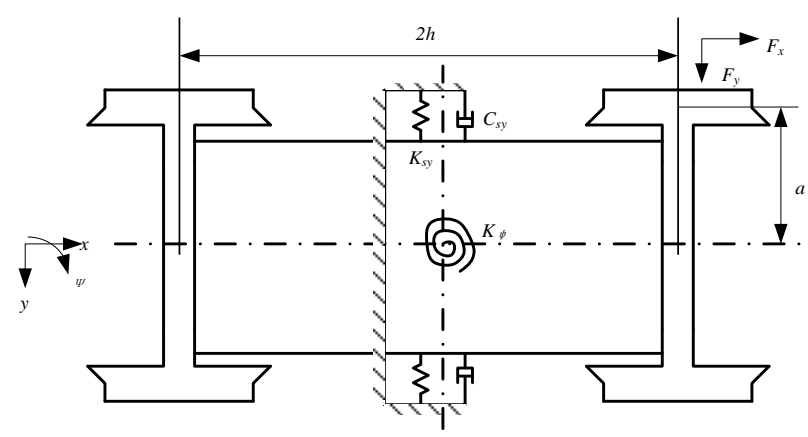

Fig. 4 Sketch of the bogie model

The equation of motion of the bogie can be defined by:

$$
\begin{gathered}
m_{\mathrm{b}} \ddot{Y}_{\mathrm{b}}=-2 C_{\mathrm{sy}} \dot{Y}_{\mathrm{b}}-2 K_{\mathrm{s} y} Y_{\mathrm{b}}-2\left(F_{y 1}+F_{y 2}\right)-F_{\mathrm{T}}, \\
I_{\mathrm{b}} \ddot{\varphi}_{\mathrm{b}}=-K_{\varphi} \varphi_{\mathrm{b}}-2 h\left(F_{y 1}-F_{y 2}\right)-2 a\left(F_{x 1}+F_{x 2}\right)-h F_{\mathrm{T}},
\end{gathered}
$$

where: $Y_{\mathrm{b}}$ and $\varphi_{\mathrm{b}}$ are the lateral and yaw motions respectively; $m_{\mathrm{b}}$ and $I_{\mathrm{b}}$ represent the mass and moment of inertia of the bogie respectively; $K_{\mathrm{s} y}$ and $C_{\mathrm{s} y}$ denote the stiffness and damping of the secondary suspension respectively; $K_{\varphi}$ is the anti-yaw stiffness; $h$ means half of wheelbase; $a$ is half of gauge; $F_{y i}$ and $F_{x i}$ mean the lateral and longitudinal wheelrail creep forces and $F_{\mathrm{T}}$ is the flange force. The specific equations of the wheel-rail force and the flange force can be found in [18].

In this TDOF bogie model, three suspension parameters, $K_{\mathrm{sy}}, C_{\mathrm{sy}}$ and $K_{\varphi}$ are considered to be random. The nominal values of those parameters in this model are listed in Table 1 and CoVs of the random parameters are set to be 10\%. Their Gaussian distributions and approximations can be expressed as:

$$
\begin{aligned}
& K_{\varphi}\left(x_{1}\right)=\sum_{i=0}^{N} \hat{k}_{\varphi i} H_{i}\left(x_{1}\right)=\mu_{k \varphi}+\sigma_{k \varphi} x_{1}, \\
& K_{\mathrm{sy}}\left(x_{2}\right)=\sum_{i=0}^{N} \hat{k}_{s y i} H_{i}\left(x_{2}\right)=\mu_{k s y}+\sigma_{k s y} x_{2}, \\
& C_{\mathrm{sy}}\left(x_{3}\right)=\sum_{i=0}^{N} \hat{k}_{c y i} H_{i}\left(x_{3}\right)=\mu_{c s y}+\sigma_{c s y} x_{3} .
\end{aligned}
$$

In order to acquire the critical speed, the speed is decreased with time $t$ in the process of the simulation. It can be defined by:

$$
V=V_{0}-t / \Delta f
$$

where: $\triangle f$ is a threshold value, herein $\Delta f=10$. 
Table 1

Parameters of the TDOF bogie model

\begin{tabular}{|c|c|c|}
\hline Notation & Designation & Value \\
\hline$m_{\mathrm{b}}$ & Bogie mass/kg & 4,963 \\
\hline$I_{\mathrm{b}}$ & Moment of inertia of bogie $/ \mathrm{kg} \cdot \mathrm{m}^{2}$ & 8,135 \\
\hline$h$ & Half of wheelbase/m & 1.5 \\
\hline$a$ & Half of gauge/m & 0.7175 \\
\hline$K_{\mathrm{s} y}$ & $\begin{array}{l}\text { Lateral stiffness of secondary suspen- } \\
\text { sion/MN/m }\end{array}$ & 0.1823 \\
\hline$C_{\mathrm{s} y}$ & $\begin{array}{l}\text { Lateral damping of secondary suspen- } \\
\qquad \operatorname{sion} / \mathrm{kN} \cdot \mathrm{s} / \mathrm{m}\end{array}$ & 29.2 \\
\hline$K_{\varphi}$ & Anti-yaw stiffness / MN/m & 2.71 \\
\hline
\end{tabular}

It is difficult to build a stochastic model by SGM due to the uncorrelation between the critical speed and the time. Hence, SCM and LHS are more suitable for the stochastic investigation of the critical speed. The numerical result without considering random parameters is given in Fig. 5 a. It can be seen that the lateral displacement of the bogie converges to a value of $0 \mathrm{~mm}$ when the running speed declines to $64.82 \mathrm{~m} / \mathrm{s}$, which is the so-called critical speed. When the random parameters are involved, the values of the critical speeds display a distribution instead of a deterministic value. Results from SCM with 2 nodes in each dimension and LHS with 500 and 20,000 samples are shown in Fig. 5 b respectively. Results show that SCM could acquire a good result which is in line with the result of LHS with 20,000 samples, while the result of LHS with 500 samples is the worst result. It strengths the conclusion that SCM exhibits the higher efficiency and accuracy than LHS.

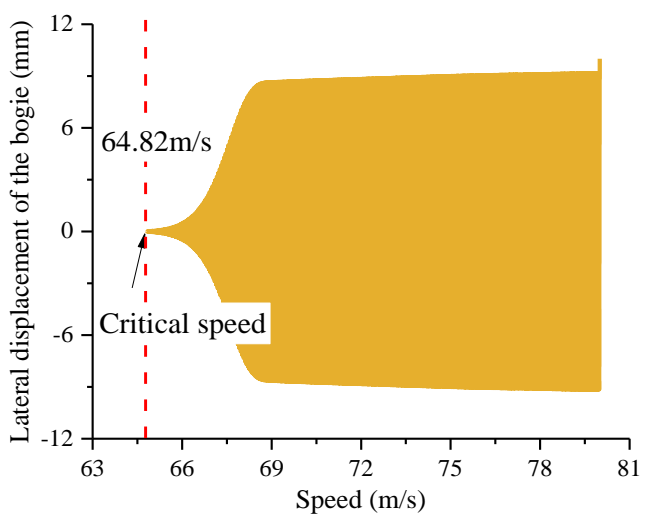

a

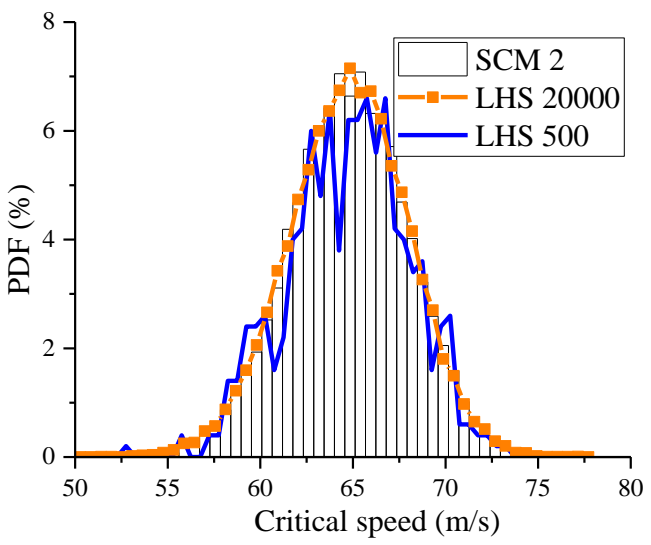

b

Fig. 5 Lateral displacement of the bogie varying with the running speed (a) and comparison of PDF of critical speeds between SCM and LHS (b)
A benchmark of LHS is made to further demonstrate the accuracy of SCM, in Fig. 6, in terms of means and Stds of critical speeds. It can be observed that both means and Stds of critical speeds show a quite stable state even though the number of nodes in SCM is small, while they vary clearly with the increase of samples in LHS. Actually, only LHS with a large number of samples can get a stable result. It is proved that the performance of SCM is prior to LHS.

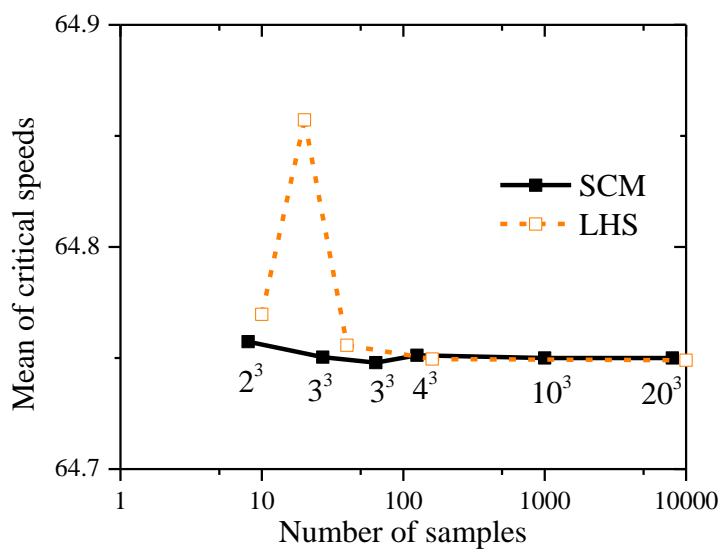

a

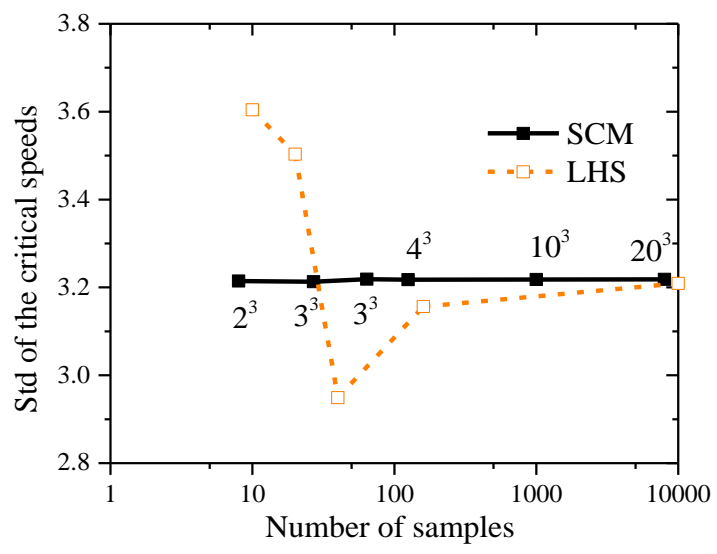

b

Fig. 6 Comparison of (a) means and (b) Stds of critical speeds between SCM and LHS

\section{MDOF vehicle-track coupled model}

As analysed in section 4, SCM has high efficiency and accuracy in the solving process of models. In this section, SCM is used in a sophisticated multi-degree-of-freedom (MDOF) vehicle-track coupled model. The deterministic MDOF vehicle-track coupled dynamics model [19 21] shown in Figure 7 includes a vehicle subsystem, a track subsystem and a nonlinear wheel-rail contact.

\subsection{Vertical vehicle-track coupled model}

Based on the structure of vehicles, the vehicle subsystem is treated as a 10-DOF multi-body submodel. The vehicle body takes vertical motion $Z_{c}$ and pitching motion $\beta_{\mathrm{c}}$ into account, and the bogie considers vertical motion $Z_{\mathrm{t}}$ and pitching motion $\beta_{\mathrm{t}}$, while the wheelset only considers the vertical motion $Z_{\mathrm{w}}$. Thus, the equation of motion for the vehicle model can be defined by: 
$\mathbf{M}_{\mathrm{v}} \ddot{\mathbf{u}}_{\mathrm{v}}+\mathbf{C}_{\mathrm{v}} \dot{\mathbf{u}}_{\mathrm{v}}+\mathbf{K}_{\mathrm{v}} \mathbf{u}_{\mathrm{v}}=\mathbf{F}_{\mathrm{v}}$,

where: $\mathbf{M}_{\mathrm{v}}, \mathbf{K}_{\mathrm{v}}$ and $\mathbf{C}_{\mathrm{v}}$ are matrices of generalized mass, stiffness and damping of vehicles respectively; $\mathbf{F}_{\mathrm{v}}$ denotes the force vector of the vehicle subsystem; $\mathbf{u}_{\mathrm{v}}$ means the displacement vector of vehicles. Corresponding parameters are listed in Table 2.

$\mathbf{u}_{v}=\left[Z_{\mathrm{c}}, \beta_{\mathrm{c}}, Z_{\mathrm{t} 1}, \beta_{\mathrm{t} 1}, Z_{\mathrm{t} 2}, \beta_{\mathrm{t} 2}, Z_{\mathrm{w} 1}, Z_{\mathrm{w} 2}, Z_{\mathrm{w} 3}, Z_{\mathrm{w} 4}\right]$

Table 2

Nominal values of the vehicle-track couple system

\begin{tabular}{|c|l|c|}
\hline Symbol & \multicolumn{1}{|c|}{ Designation } & Mean \\
\hline$M_{\mathrm{c}}$ & Car body mass $/ \mathrm{kg}$ & 46,060 \\
\hline$M_{\mathrm{t}}$ & Bogie mass $/ \mathrm{kg}$ & 2,235 \\
\hline$M_{\mathrm{w}}$ & Wheelset mass $/ \mathrm{kg}$ & 1,457 \\
\hline$J_{\mathrm{c}}$ & Moment of inertia of car body $/ \times 10^{6} \mathrm{~kg} \cdot \mathrm{m}^{2}$ & 1.95 \\
\hline$J_{\mathrm{t}}$ & Moment of inertia of bogie $/ \mathrm{kg} \cdot \mathrm{m}^{2}$ & 1,238 \\
\hline$K_{\mathrm{p} z}$ & Stiffness of primary suspension $/ \mathrm{MN} / \mathrm{m}$ & 1.773 \\
\hline$K_{\mathrm{s} z}$ & Stiffness of secondary suspension $/ \mathrm{MN} / \mathrm{m}$ & 0.8 \\
\hline$C_{\mathrm{p} z}$ & Damping of primary suspension $/ \mathrm{kN} \cdot \mathrm{s} / \mathrm{m}$ & 20 \\
\hline$C_{\mathrm{s} z}$ & $\begin{array}{l}\text { Damping of secondary suspension } / \\
\text { kN } / \mathrm{s} / \mathrm{m}\end{array}$ & 120 \\
\hline$L_{\mathrm{c}}$ & Half of length between truck centres $/ \mathrm{m}$ & 8.6875 \\
\hline$L_{\mathrm{t}}$ & Half of wheelbase $/ \mathrm{m}$ & 1.25 \\
\hline$g$ & Acceleration of gravity $/ \mathrm{m} \cdot \mathrm{s}^{-2}$ & 9.8 \\
\hline$v$ & Vehicle speed $/ \mathrm{km} / \mathrm{h}$ & 300 \\
\hline$m_{\mathrm{r}}$ & Rail mass per meter $/ \mathrm{kg}$ & 60.64 \\
\hline$M_{\mathrm{s}}$ & Half of Sleeper mass $/ \mathrm{kg}$ & 125.5 \\
\hline$M_{\mathrm{b}}$ & Ballast mass $/ \mathrm{kg}$ & 310 \\
\hline$K_{\mathrm{p}}$ & Rail pad stiffness $/ \mathrm{kN} / \mathrm{mm}$ & 60 \\
\hline$C_{\mathrm{p}}$ & Rail pad damping $/ \mathrm{kN} \cdot \mathrm{s} / \mathrm{m}$ & 75 \\
\hline$K_{\mathrm{b}}$ & Ballast stiffness $/ \mathrm{kN} / \mathrm{mm}$ & 150 \\
\hline$C_{\mathrm{b}}$ & Ballast damping $/ \mathrm{kN} \cdot \mathrm{s} / \mathrm{m}$ & 60 \\
\hline$K_{\mathrm{f}}$ & Subgrade elastic stiffness $/ \mathrm{kN} / \mathrm{mm}$ & 70 \\
\hline$C_{\mathrm{f}}$ & Subgrade damping $/ \mathrm{kN} \cdot \mathrm{s} / \mathrm{m}$ & 100 \\
\hline$L_{\mathrm{s}}$ & Sleeper space $/ \mathrm{m}$ & 0.6 \\
\hline$E I$ & Flexural rigidity of rails $/ \mathrm{MN} \cdot \mathrm{m}^{2}$ & 6.6 \\
\hline & & \\
\hline
\end{tabular}

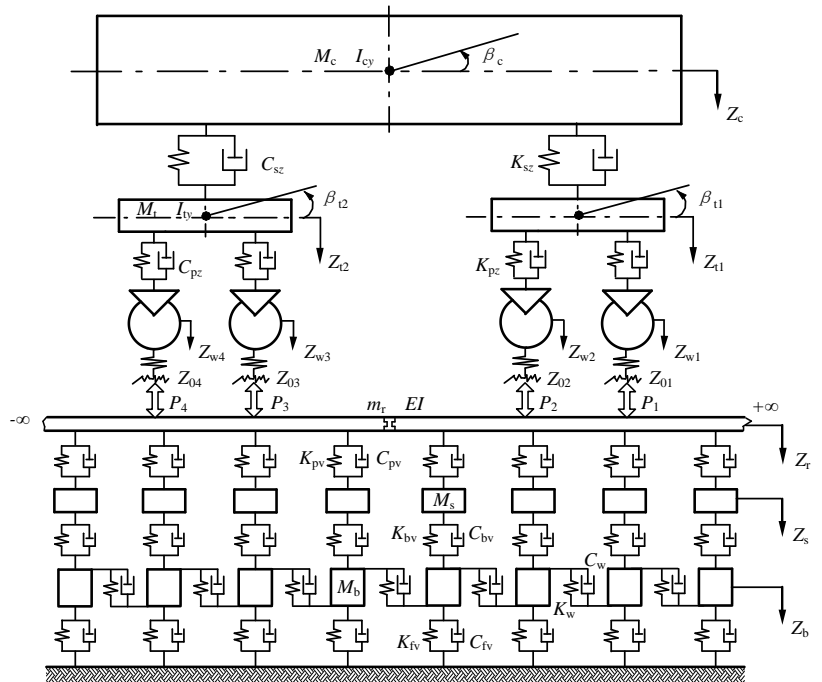

Fig. 7 Side view of the MDOF vehicle-track coupled model

In generally, the ballasted track consists of three components, i.e., the rail, sleepers and ballast. The rail is modelled as finite Euler-Bernoulli beams. Only vertical vibrations are taken into account for all components. The equations of motion of the track subsystem are expressed as:
$\mathbf{M}_{\mathrm{t}} \ddot{\mathbf{u}}_{\mathrm{t}}+\mathbf{K}_{\mathrm{t}} \mathbf{u}_{\mathrm{t}}+\mathbf{C}_{\mathrm{t}} \dot{\mathbf{u}}_{\mathrm{t}}=\mathbf{F}_{\mathrm{t}}$,

where: $\mathbf{M}_{t}, \mathbf{K}_{\mathrm{t}}$ and $\mathbf{C}_{\mathrm{t}}$ are matrices of generalized mass, stiffness and damping of track subsystem respectively; $\mathbf{F}_{\mathrm{t}}$ is the vector of force of track subsystem; and $\mathbf{u}_{\mathrm{t}}$ is the vector of displacement of track subsystem. The track parameters used in this part are listed in Table 2.

It is assumed that suspension parameters of vehicles are provided with a certain level of working tolerance fitting different distributions. In detail, Gaussian distributions with zero correlation are independent and CoVs are below $15 \%$. In this stochastic model, the random parameters are generated as samples for the vehicle-track system. In this way, a stochastic model is expressed as:

$$
\left\{\begin{array}{c}
\mathbf{M}_{\mathrm{v}} \ddot{\mathbf{u}}_{\mathrm{v}}\left(t, \mathbf{Z}_{\mathrm{v}}, \mathbf{Z}_{\mathrm{t}}\right)+\mathbf{K}_{\mathrm{v}} \mathbf{u}_{\mathrm{v}}\left(t, \mathbf{Z}_{\mathrm{v}}, \mathbf{Z}_{\mathrm{t}}\right)+\mathbf{C}_{\mathrm{v}} \dot{\mathbf{u}}_{\mathrm{v}}\left(t, \mathbf{Z}_{\mathrm{v}}, \mathbf{Z}_{\mathrm{t}}\right)=\mathbf{F}_{\mathrm{v}} \\
\mathbf{M}_{\mathrm{t}} \ddot{\mathbf{u}}_{\mathrm{t}}\left(t, \mathbf{Z}_{\mathrm{v}}, \mathbf{Z}_{\mathrm{t}}\right)+\mathbf{K}_{\mathrm{t}} \mathbf{u}_{\mathrm{t}}\left(t, \mathbf{Z}_{\mathrm{v}}, \mathbf{Z}_{\mathrm{t}}\right)+\mathbf{C}_{\mathrm{t}} \dot{\mathbf{u}}_{\mathrm{t}}\left(t, \mathbf{Z}_{\mathrm{v}}, \mathbf{Z}_{\mathrm{t}}\right)=\mathbf{F}_{\mathrm{t}}
\end{array},\right.
$$

where: $\mathbf{Z}_{\mathrm{v}}$ and $\mathbf{Z}_{\mathrm{t}}$ represent the random variables of vehicles and track respectively.

\subsection{Uncertainty quantification analysis of dynamic re-} sponses under a track irregularity

In addition to rail defects, track irregularities are common excitations for the vehicle-track system. Accordingly, the influence of the random parameters on the vehicle dynamics under a track irregularity is discussed in this section.

A ballasted track spectrum of the vertical irregularity for high-speed railway lines has been defined as power functions by China Railway Corporation. Specific coefficients of power functions are shown in Fig. 8. A sample of the vertical irregularity can be acquired by the inverse transformation of the track spectrum. In this study, only the suspension parameters are set to be random in the stochastic analysis. To be specific, four random parameters, i.e., $K_{\mathrm{pz}}$, $C_{\mathrm{pz}}, K_{\mathrm{sz}}$, and $C_{\mathrm{sz}}$, are considered to follow Gaussian distributions with CoVs of $10 \%, 20 \%$ and $30 \%$. SCM is employed to carry out the uncertainty quantification analysis for the stochastic vehicle-track coupled model.

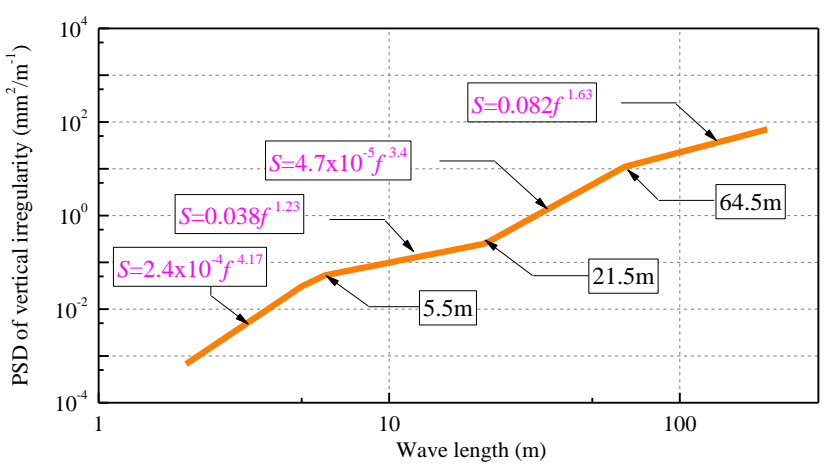

Fig. 8 Ballasted track spectrum

The qualification of interest is limited to the vertical acceleration of vehicle body and the Sperling index, which are important assessment criteria for the running quality. As an example, Fig. 9 gives the PDF evolution of vehicle body acceleration with respect to time in the case of $\mathrm{CoV}=10 \%$, where the peaks refer to the probabilities of accelerations. 


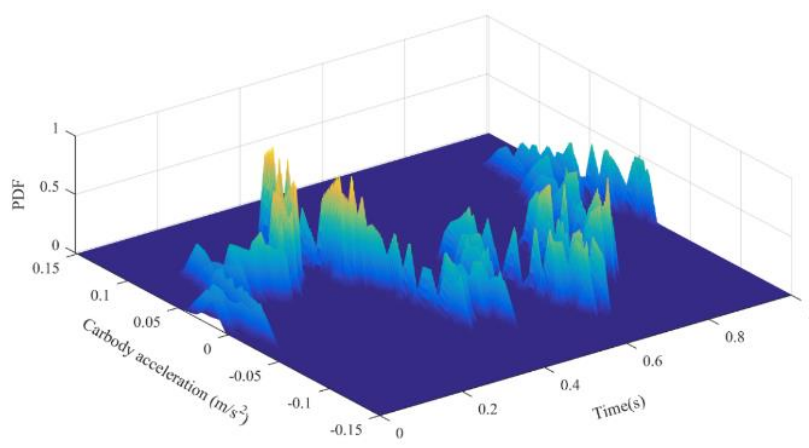

Fig. 9 PDF evolution of vertical vehicle body acceleration in time domain

The Sperling index can be used to assess the riding performance, which is defined by:

$$
W=7.08\left[\frac{A^{3}}{f} F(f)\right]^{0.1},
$$

where: $A$ is the vehicle body acceleration amplitude at the frequency $f ; F(f)$ is a weighted coefficient depending on the frequency $f$. There are three levels of riding performance for high speed trains, i.e., first level: $W<2.5$; second level: $2.5 \leq W<2.75$; and third level: $2.75 \leq W<3$. Generally, the running quality of new vehicles should meet the requirement of the first level at least.

Based on the limit standard of the first level, the influence of parametric uncertainties on Sperling index is studied. Fig. 10 shows the probability distributions of Sperling index under the influence of the various CoVs. It can be seen that Sperling indices roughly fit Gaussian distributions and the dispersion degrees become larger with the rise of CoV. To be specific, with CoVs of input parameters increasing from $10 \%$ to $30 \%$, the corresponding Stds rise from 0.06 to 0.16 , and the probabilities of the over-limit for Sperling index (>2.5) increase from $1.87 \%$ to $27.74 \%$. It reflects the fact that the random parameters should be considered in the assessment of the riding performance.

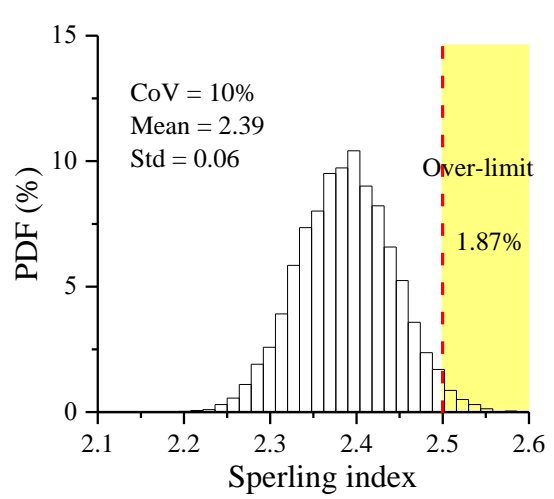

a

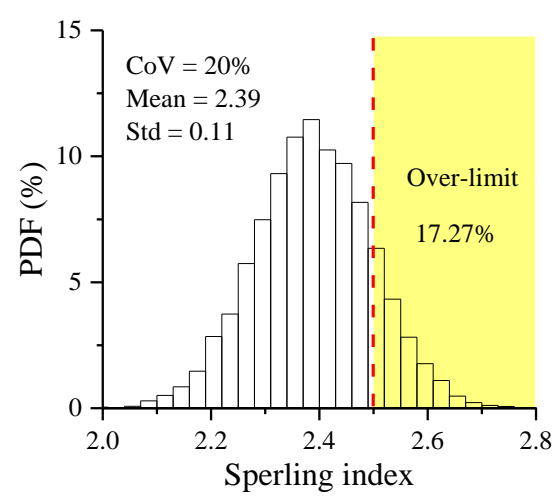

b

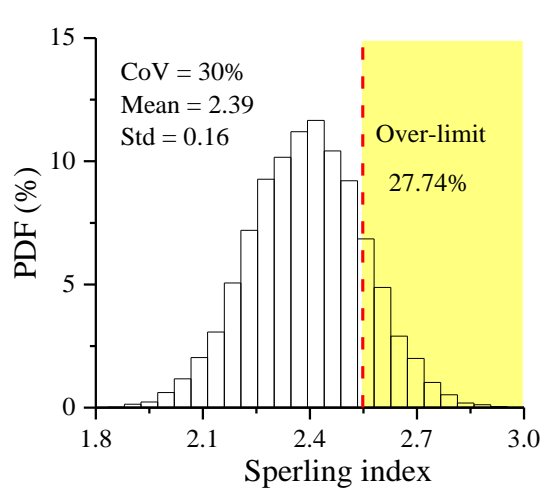

$\mathrm{c}$

Fig. 10 PDF of the Sperling index under different CoVs of random input parameters: $\mathrm{CoV}=10 \%(\mathrm{a}) ; \mathrm{CoV}=20 \%(\mathrm{~b})$; and $\mathrm{CoV}=30 \%$ (c)

\section{Conclusions}

This paper shows how uncertainties in railway vehicle-track system influences the vehicle dynamics. Three approaches are to clarify the propagation of uncertainties during the whole process, beginning with the parameters input in the vehicle-track mathematical model and ending with the results of running dynamics by means of SGM, SCM and LHS. Firstly, a stochastic SDOF wheel model has been built to illustrate the implements of SGM, SCM and LHS. Then, a simplified stochastic TDOF bogie model has been established to analyse the critical speed with three random input parameters, where solving method adopts SCM and LHS respectively. At last, a stochastic vertical vehicletrack model has been well-built based on SCM, where impact behaviour of wheel-rail interaction under a rail defect is investigated and dynamic response of vehicles under a track irregularity is analysed in terms of the Sperling index. Through above analysis, following conclusions can be drawn:

1. In terms of the efficiency and accuracy, the performance of SCM is better than that of SGM and LHS, due to the easy implementation of SCM.

2. In the TDOF bogie model, the uncertainties of suspension parameters give rise to an obvious influence on the critical speed and should be attached enough importance in the design process of bogies.

3. As for the running quality, a higher $\mathrm{CoV}$ of given distributions leads to a larger probability of the overlimit (>2.5) for Sperling index.

\section{Acknowledgement}

This work was supported by the National Natural Science Foundation of China (Grant No. 71801019) and the Fundamental Research Funds for the Central Universities, CHD (Grant No. 300102218116 and Grant No. 300102229106).

\section{References}

1. Funfschilling, C.; Perrin, G.; Kraft, S. 2012. Propagation of variability in railway dynamic simulations: application to virtual homologation, Veh. Syst. Dyn. 50(sup1): 245-261. https://doi.org/10.1080/00423114.2012.676757.

2. Sandu, A.; Sandu, C.; Ahmadian, M. 2006. Modeling multibody systems with uncertainties. Part I: Theoretical and computational aspects, Multibody Syst. Dyn. 15(4): 369-391. https://doi.org/10.1007/s11044-006-9007-5.

3. Sandu, C.; Sandu, A.; Ahmadian, M. 2006. Modeling 
multibody systems with uncertainties. Part II: Numerical applications, Multibody Syst. Dyn. 15(3): 241-262. https://doi.org/10.1007/s11044-006-9008-4.

4. Kewlani, G.; Crawford, J.; Iagnemma, K. 2012. A polynomial chaos approach to the analysis of vehicle dynamics under uncertainty, Veh. Syst. Dyn. 50(5): 749774.

https://doi.org/10.1080/00423114.2011.639897.

5. Wu, J.; Luo, Z.; Zhang, N.; Zhang, Y. 2015. A new uncertain analysis method and its application in vehicle dynamics, Mech. Syst. Signal Process. 50-51: 659-675. https://doi.org/10.1016/j.ymssp.2014.05.036.

6. Wu, J.; Luo, Z.; Zhang, N.; Zhang, Y. 2016. Dynamic computation of flexible multibody system with uncertain material properties, Nonlinear Dyn. 85(2): 12311254. https://doi.org/10.1007/s11071-016-2757-6.

7. Oscarsson, J. 2002. Simulation of train-track interaction with stochastic track properties, Veh. Syst. Dyn. 37(6): 449-469. https://doi.org/10.1076/vesd.37.6.449.3521.

8. Oscarsson, J. 2002. Dynamic train-track-ballast interaction with unevenly distributed track properties, Veh. Syst. Dyn. 37(sup1): 385-396. https://doi.org/10.1080/00423114.2002.11666248

9. Li, M.X.D.; Berggren, E.G. 2010. A study of the effect of global track stiffness and its variations on track performance: Simulation and measurement, P. I. Mech. Eng. F-J. Rai. 224(5): 375-382. https://doi.org/10.1243/09544097jrrt361.

10. Funfschilling, C.; Perrin, G.; Sebes, M.; Bezin, Y.; Mazzola, L.; Tajan, M.L.N. 2015. Probabilistic simulation for the certification of railway vehicles, P. I. Mech. Eng. F-J. Rai. 229(6): 770-781. https://doi.org/10.1177/0954409715589395.

11. Bigoni, D.; True, H.; Engsig-Karup, A.P. 2014. Sensitivity analysis of the critical speed in railway vehicle dynamics, Veh. Syst. Dyn. 52(sup1): 272-286. https://doi.org/10.1080/00423114.2014.898776.

12. Perrin, G.; Duhamel, D.; Soize, C.; Funfschilling, C. 2015. Quantification of the influence of the track geometry variability on the train dynamics, Mech. Syst. Signal Process. 60-61: 945-957. https://doi.org/10.1016/j.ymssp.2015.01.004.

13. Lestoille, N.; Soize, C.; Funfschilling, C. 2016. Sensitivity of train stochastic dynamics to long-term evolution of track irregularities, Veh Syst Dyn. 54(5): 545-567. https://doi.org/10.1080/00423114.2016.1142095.

14. Xu, L.; Zhai, W. 2017. A new model for temporal-spatial stochastic analysis of vehicle-track coupled systems, Veh. Syst. Dyn. 55(3): 427-448. https://doi.org/10.1080/00423114.2016.1270456.

15. Xu, L.; Zhai, W.; Gao, J.; Meacci, M.; Chen, X. 2017. On effects of track random irregularities on random vibrations of vehicle-track interactions, Probabilist Eng. Mech. 50: 25-35. https://doi.org/10.1016/j.probengmech.2017.10.002.

16. Xiu, D. 2010. Numerical methods for stochastic computations: a spectral method approach. Princeton: Princeton University Press. 125 p.

17. Le, M.O.P.; Knio, O.M. 2010. Spectral methods for uncertainty quantification: with applications to computational fluid dynamics, Dordrecht: Springer. $536 \mathrm{p}$.
18. True, H.; Kaas-Petersen, C. 1983. A bifurcation analysis of nonlinear oscillations in railway vehicles, Veh. Syst. Dyn. 12(1-3): 5-6. https://doi.org/10.1080/00423118308965288.

19. Zhai, W.; Sun, X. 1994. A detailed model for investigating vertical interactions between railway vehicle and track, Veh. Syst. Dyn. 23(sup1): 603-615. https://doi.org/10.1080/00423119308969544.

20. Zhai, W.; Cai, C.; Guo, S. 1996. Coupling model of vertical and lateral vehicle/track interactions, Veh. Syst. Dyn. 26(1): 61-79. https://doi.org/10.1080/00423119608969302.

21. Zhai, W.; Wang, K.; Cai, C. 2009. Fundamentals of vehicle-track coupled dynamics. Veh. Syst. Dyn. 47(11): 1349-1376. https://doi.org/10.1080/00423110802621561.

\section{Zhang, P. Xu, D. Bigoni}

\section{APPLICATION AND COMPARISON OF UNCERTAINTY QUANTIFICATION METHODS FOR RAILWAY VEHICLE DYNAMICS WITH RANDOM MECHANICAL PARAMETERS}

S u m m a r y

This paper aims to investigate uncertainties in railway vehicle suspension components and the implement of uncertainty quantification methods in railway vehicle dynamics. The sampling-based method represented by Latin Hypercube Sampling (LHS) and generalized polynomial chaos approaches including the stochastic Galerkin and Collocation methods (SGM and SCM) are employed to analyze the propagation of uncertainties from the parameters input in a vehicle-track mathematical model to the results of running dynamics. In order to illustrate the performance qualities of SGM, SCM and LHS, a stochastic wheel model with uncertainties of the stiffness and damping is firstly formulated to study the vertical displacement of wheel. Numerical results show that SCM, which can be easily implemented by means of the existing deterministic model, has explicit advantages over SGM and LHS in terms of the efficiency and accuracy. Furthermore, a simplified stochastic bogie model with three random suspension parameters is also established by means of SCM and LHS to analyze the critical speed, which is affected obviously by the parametric uncertainties. Finally, a stochastic vertical vehicle-track coupled model with parametric uncertainties is built comprehensively on the basis of SCM, by which the dynamic response of vehicles under the track irregularity is explored in terms of the Sperling index. It concludes that the uncertainties of parameters have a significant influence on Sperling index from the view of the running quality.

Keywords: uncertainty quantification; railway vehicle dynamics; sampling-based method; polynomial chaos.

Received April 26, 2019

Accepted November 21, 2019 